\title{
Comparative studies of MSGC and GEM for photon and electron detection at ambient conditions
}

\author{
J.A. Mir $^{a, *}$, A.S. Conceição ${ }^{b}$, J.M. Maia ${ }^{\text {b,c }}$, J.F.C.A. Veloso ${ }^{\text {d }}$, J.M.F. dos Santos ${ }^{b}$ \\ a Science and Technology Facilities Council, Rutherford Appleton Laboratory, Chilton, Didcot, Oxon OX11 0QX, UK \\ b Instrumentation Center, Physics Department, University of Coimbra, 3004-516 Coimbra, Portugal \\ ${ }^{c}$ Physics Department, University of Beira Interior, 6201-001 Covilhã, Portugal \\ d Physics Department, University of Aveiro, 3810-193 Aveiro, Portugal
}

\section{A R T I C L E I N F O}

Article history:

Received 23 May 2008

Received in revised form

17 June 2008

Accepted 25 September 2008

Available online 10 October 2008

Keywords:

MSGC

GEM

X-ray absorption spectroscopy

Total electron yield

Fluorescent yield

\begin{abstract}
A B S T R A C T
Experimental work relevant to the electron and photon detection in X-ray Absorption Spectroscopy (XAS) experiments is described using a Micro-Strip Gas Chamber (MSGC) and a Gas Electron Multiplier (GEM) operated at atmospheric pressure. XAS studies at ambient conditions dictate the use of predominately Helium counter gas mixture with minimal quench gas concentration whilst attaining charge gains in the $10^{4}$ region. We present results on the charge gain for both MSGC and GEM detectors using He/iso- $\mathrm{C}_{4} \mathrm{H}_{10}$ based gas mixtures. Pulse-height distributions due to the Total Electron Yield (TEY) arising from interactions between $8.05 \mathrm{keV}$ X-rays and Titanium sample, i.e. photoelectrons, auger electrons and fluorescent photons, are also presented. Employing a repelling voltage grid close to the sample surface permitted the separation of TEY and the Fluorescent Yield (FY) signal.
\end{abstract}

(c) 2008 Elsevier B.V. All rights reserved.

\section{Introduction}

Introduced by Oed [1] the Micro-Strip Gas Counter (MSGC) is realised on a plate of some suitable semi-insulating material using microlithography techniques. Fine anode strips (typically $10 \mu \mathrm{m}$ wide) are interleaved with cathode strips (typically $>100 \mu \mathrm{m}$ wide) in a repetitive pattern which has a pitch of several hundred microns. When a voltage of about $\approx 500 \mathrm{~V}$ is established between anode and cathode strips in a suitable gas atmosphere the free electrons deposited in the gas experience amplification near the anode strips. These signals can be detected on individual anodes or on areas of bussed anode strips.

The gas electron multiplier (GEM) was invented at CERN in 1997 [2] and consists of a thin polymer mesh $(50 \mu \mathrm{m}$ thick Kapton), metal clad on both sides usually by $5 \mu \mathrm{m}$ thick copper and perforated with a high density of holes (typically $40-120 \mu \mathrm{m}$ hole diameter, $140-200 \mu \mathrm{m}$ hole pitch). Upon the application of a potential difference across the GEM holes in a suitable counter gas mixture, charge amplification is achieved when electrons enter the GEM holes.

Conventional X-ray Absorption Spectroscopy (XAS) uses ionisation chambers to monitor signal attenuation by a sample as a

\footnotetext{
* Corresponding author. Tel.: +441235446262; fax: +441235446863.

E-mail address: J.A.Mir@rl.ac.uk (J.A. Mir).
}

function of the photon energy. The absence of the charge gain in ionisation chambers renders the conventional XAS studies rather disadvantageous for applications where the sample contains low atomic species (Carbon, Nitrogen, Oxygen, etc.) or when studying the transition metal oxides involving LM transitions for which a detector response in the $270-1000 \mathrm{eV}$ X-ray energy region is required. To improve the conventional XAS at lower X-ray energies requires the introduction of proportional counters $[3,4]$. In the present study, we have used a MSGC and a GEM to study the pulse-height distributions resulting from the emissions caused by de-excitations of a Titanium sample excited by a laboratory based $\mathrm{X}$-ray source ( $8.05 \mathrm{keV} \mathrm{Cu}-\mathrm{K} \mathrm{X}$-rays). For XAS experiments utilising the gaseous detector technology, it becomes necessary to use predominately Helium in the counter gas mixtures. This allows the passage of the primary incoming beam to interact with the sample atoms without distorting the output signal due to interactions with the counter gas and by other side minimises the pulse-height spectra distortion due to the re-absorption of primary electrons deposited by the fluorescent photons of low energy in the gas medium close to the sample/drift-electrode.

In order to accomplish sub-keV X-rays detection, the detector must achieve a charge gain of $\sim 10^{4}$ with the Helium based gas mixtures. In the present studies, the charge gain characteristics of a MSGC were examined as a function of its anode-cathode voltage using $\mathrm{He} /$ iso- $\mathrm{C}_{4} \mathrm{H}_{10}$ (75/25\%) and $\mathrm{He} /$ iso- $\mathrm{C}_{4} \mathrm{H}_{10}$ (85/15\%) whilst similar work was carried out to establish the charge gain 
characteristics of a GEM as a function of voltage applied across the GEM holes for a number of different $\mathrm{He} /$ iso- $\mathrm{C}_{4} \mathrm{H}_{10}$ gas mixtures.

Total Electron Yield (TEY) XAS technique combined with gaseous detectors offers the possibility of investigating the surfaces at ambient conditions [5-8]. The TEY method is particularly suited for obtaining quantitative information about the local atomic structure of diluted atomic species in solids as well as liquids. The interaction of a synchrotron beam with a given sample, usually at glancing angles, produces photoelectrons, auger electrons and fluorescent photons. The primary charge signal is amplified by the gaseous detector thereby improving the signal-to-noise ratio. In order to examine the X-ray absorption coefficient around the constituent $\mathrm{K}$, L or M absorption edges, the energy of the incoming photon is varied using a monochromator.

Whilst TEY is a surface phenomena, typically $10-100 \mathrm{~nm}$, the fluorescent photons produced by the incoming X-ray beam emerge from the bulk (several microns deep) of the material being examined. These are usually absorbed within the active volume of the gaseous detector and constitute the Fluorescent Yield (FY) signal. In order to study TEY and FY independently requires a repelling voltage grid in the proximity of the sample [9]. By biasing the repelling grid negative with respect to the sample surface, the signal due to the photoelectron and auger electrons (TEY) is almost entirely blocked permitting only the fluorescent events (FY) to be recorded. In the present studies we will demonstrate the potential of this concept using a Titanium sample.

\section{Experimental setup and methodology}

The experimental arrangement for measuring the pulse-height distributions and charge gains of the MSGC and GEM is shown in Fig. 1, noting that either a MSGC or a GEM was used at any given time. The MSGC plate was manufactured by Baumer IMT, Greifensee, Switzerland using the semiconducting S8900 glass. The electrode geometry was based on a previous high charge gain structure albeit using argon based counter gas mixtures [10], and consisted of $300 \mu \mathrm{m}$ wide cathodes and $5 \mu \mathrm{m}$ anodes set at

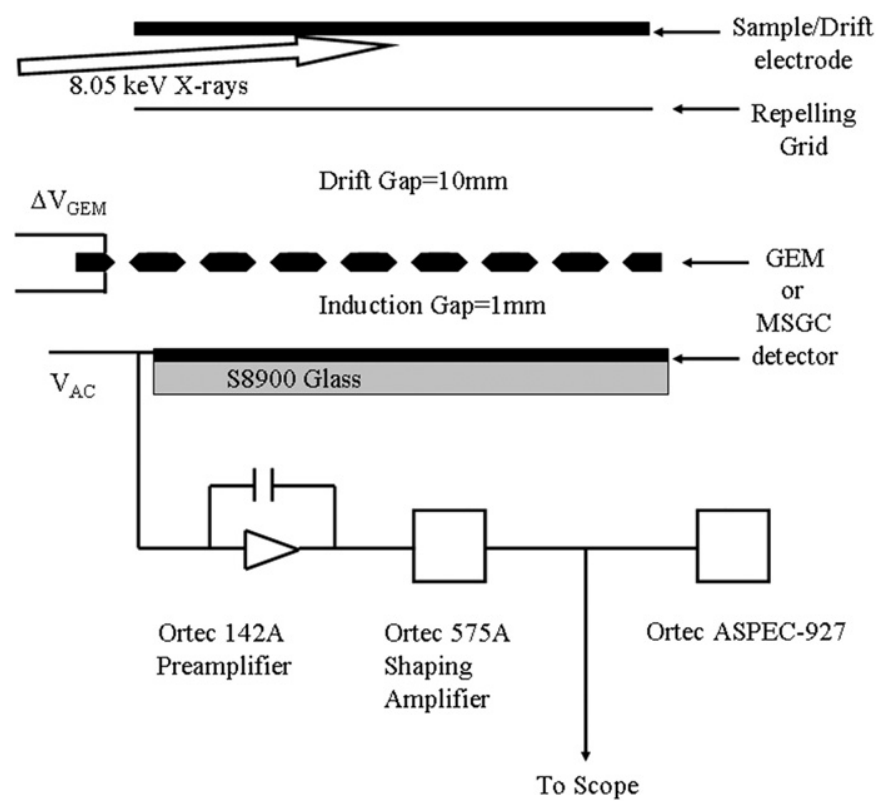

Fig. 1. Schematic arrangement showing the MSGC and the GEM detector.
$500 \mu \mathrm{m}$ pitch. The active area of the device was approximately $20 \times 10 \mathrm{~mm}^{2}$.

The GEM was fabricated at the CERN TS-DEM workshop and consisted of a $50 \mu \mathrm{m}$ thick copper clad ( $5 \mu \mathrm{m}$ thickness) Kapton foil with 70 (50) $\mu \mathrm{m}$ diameter holes in copper (Kapton) patterned at $140 \mu \mathrm{m}$ hole pitch. The GEM used here had an active area of $50 \times 50 \mathrm{~mm}^{2}$ and was supported on a G-10 frame. The gap between the GEM and the readout plane is known as the induction gap and was set at $1 \mathrm{~mm}$ whilst the drift gap (distance between sample and GEM or MSGC) was set at $10 \mathrm{~mm}$.

The first part of the present work was to determine the charge gain characteristics of the MSGC and the GEM. This was done using a Fe-55 (Mn-K X-ray (5.89 keV)) beam which illuminated the detector drift space perpendicular to the MSGC or the GEM planes. In this study, the detection chamber was operated at a constant gas flow rate where the gas composition was controlled by Brooks mass-flow controllers (model 5850E) built into a rig that was constructed using stainless steel tubing. The count rates used throughout these studies were kept in the region of $10 \mathrm{kHz}$.

For the case of MSGC, the variation of charge gain was investigated as a function of the potential difference $V_{\mathrm{ac}}$ across the MSGC anode and cathode strips. Charge gain characteristics were obtained using $\mathrm{He} /$ iso- $\mathrm{C}_{4} \mathrm{H}_{10}$ gas mixtures with concentrations of $75 / 25 \%$ and $85 / 15 \%$. The drift electrode was operated at negative potential $\left(V_{\mathrm{d}}=-1500 \mathrm{~V}\right)$ with respect to the MSGC anodes that were held either close to ground. The MSGC anodestrips were connected to an Ortec preamplifier (model 142A). The preamplifier output is then fed into an Ortec shaping amplifier (model 575A) with shaping time constants adjusted to $0.5 \mu \mathrm{s}$. Bipolar output of the shaping amplifier in turn fed an Ortec pulseheight analyser (Ortec ASPEC-927).

The charge gain characteristics of the GEM were also investigated as a function of the voltage applied across the GEM holes, $\Delta V_{\mathrm{GEM}}$, whilst the induction field was set at $6 \mathrm{kV} / \mathrm{cm}$. Four different concentrations of iso- $\mathrm{C}_{4} \mathrm{H}_{10}(5,15,20$ and $25 \%)$ in Helium were used. In each set of measurements, $\Delta V_{\mathrm{GEM}}$ was gradually increased up to the onset of micro-discharges. In order to protect the GEM from current surges, we used a twin high-voltage tracking power-supply (Iseg, NHQ 224L_503_K04) which allowed the voltages at both sides of the GEM to be ramped up simultaneously by the first channel when setting the induction field and then increasing voltage at the topside of the GEM using the second channel. The trip limit was set to $0.5 \mu \mathrm{A}$ currents, beyond which the power supplies were automatically turned off.

The charge gain for each $V_{\mathrm{ac}}$ or $\Delta V_{\mathrm{GEM}}$ used was obtained by comparing the peak of the pulse-height distribution produced by the Fe-55 radioactive source and then calibrating the pulse-height spectrum with an accurately known charge. This was done in the usual way of by supplying a known step voltage into the preamplifier test input capacitance. By dividing the peak charge with the number of primary electrons produced in the conversion/ drift region of the detector yielded the effective charge gain for a given bias settings. For example, in the case of $\mathrm{He} /$ iso- $\mathrm{C}_{4} \mathrm{H}_{10}$ $(85 / 15 \%)$ the primary charge $n_{0} \approx 154$ electron-ion pairs, taking into account an average energy $w \approx 38.3 \mathrm{eV}[11,12]$ to produce an electron-ion pair in this gas mixture.

In order to study TEY and FY independently, a repelling grid comprising of $50 \mu \mathrm{m}$ diameter nickel wires at $0.5 \mathrm{~mm}$ pitch and running in a direction parallel to the X-ray beam. This grid was held at approximately $2 \mathrm{~mm}$ from the drift (Titanium plate) electrode. A collimated X-ray generator beam (8.05 keV Cu-K $\mathrm{X}$-rays) was used to excite the Titanium sample at grazing incidence. For a given counter gas mixture and for voltages $V_{\mathrm{ac}}$ or $\Delta V_{\mathrm{GEM}}$, pulse-height distributions were recorded as a function of the repelling grid voltage, $V_{\mathrm{g}}$, whilst the drift voltage was held at $V_{\mathrm{d}}=-1500 \mathrm{~V}$. 


\section{Experimental results and discussion}

Fig. 2 shows the variation of the charge gain versus the anodecathode voltage, $V_{\mathrm{ac}}$, for the present MSGC using $\mathrm{He} / \mathrm{iso}-\mathrm{C}_{4} \mathrm{H}_{10}$ gas mixtures of $75 / 25 \%$ and $85 / 15 \%$. The maximum charge gains before the onset of electrical instabilities are approximately $2 \times 10^{4}$ and $6 \times 10^{3}$ for $\mathrm{He} /$ iso- $\mathrm{C}_{4} \mathrm{H}_{10}$ of $75 / 25 \%$ and $85 / 15 \%$, respectively, showing the importance of the quench gas concentration to achieve higher gains. We note that upper charge gains are much lower than those obtained using argon based gas mixture for similar (optimised) MSGCs [10]. Fig. 3 shows the variation of the charge gain versus the voltage applied across the GEM holes, $\Delta V_{\text {GEM }}$, for several $\mathrm{He} /$ iso- $\mathrm{C}_{4} \mathrm{H}_{10}$ gas mixtures of $95 / 5 \%$, $85 / 15 \%, 80 / 20 \%$ and $75 / 25 \%$. The induction field during these measurements was set at $6 \mathrm{kV} / \mathrm{cm}$. Fig. 3 demonstrates that the present GEM based detector can achieve charge gain exceeding $5 \times 10^{4}$ thus easily permitting studies in the sub-keV X-ray region. The high charge gain properties of the GEM, at relatively small concentration of the quench gas, are attributed to electron multiplication occurring within the GEM holes, and consequently the avalanche confinement allows a lower photon feedback [13]

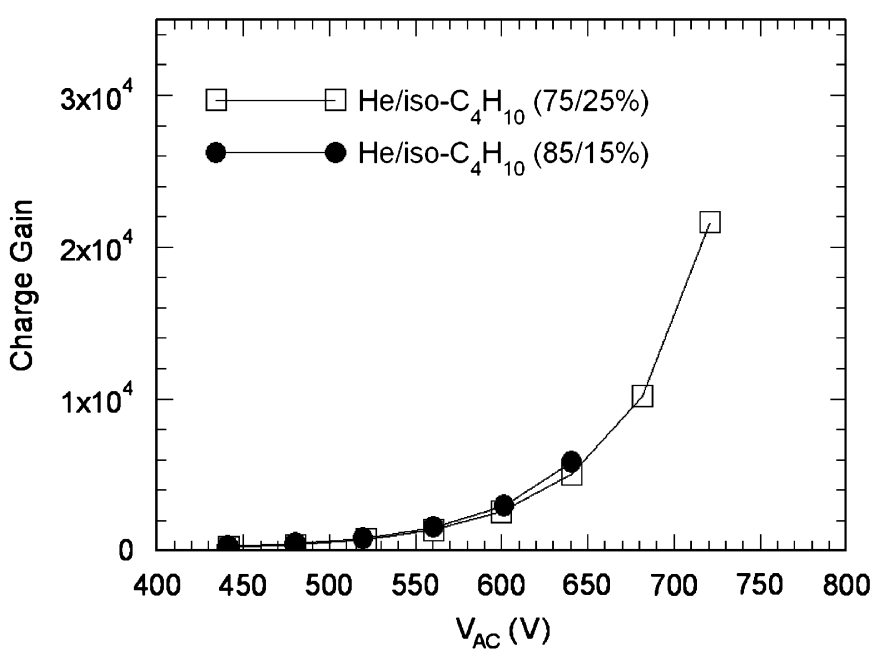

Fig. 2. Charge gain of the MSGC as a function of the anode-cathode voltage, $V_{\mathrm{ac}}$, using $\mathrm{He} /$ iso- $\mathrm{C}_{4} \mathrm{H}_{10}$ gas mixtures of $75 / 25 \%$ and $85 / 15 \%$.

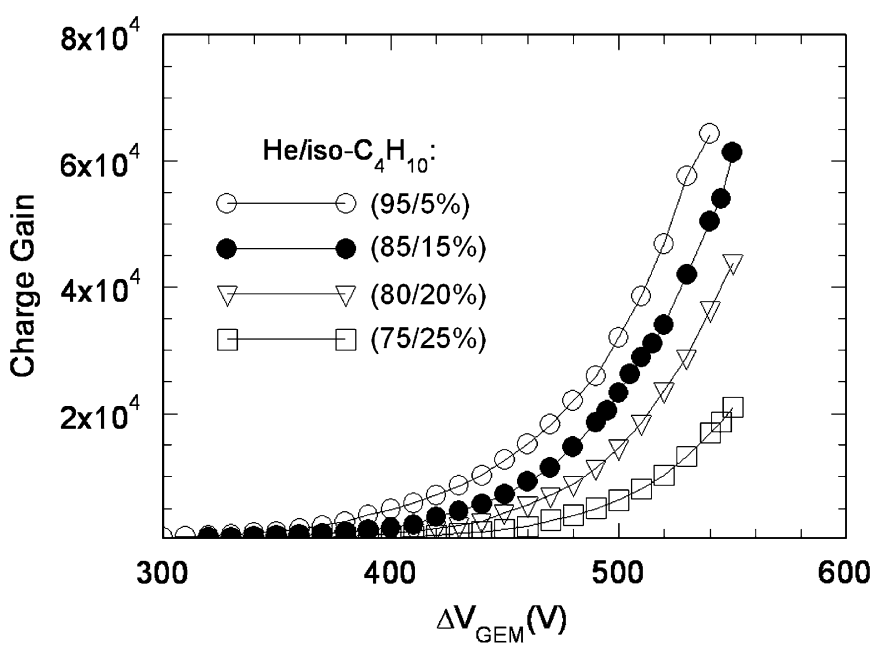

Fig. 3. Charge gain of the GEM as a function of the voltage applied across the GEM holes, $\Delta V_{\mathrm{GEM}}$, using $\mathrm{He} /$ iso- $_{4} \mathrm{H}_{10}$ gas mixtures of $95 / 5 \%, 85 / 15 \%, 80 / 20 \%$ and $75 / 25 \%$. and improved detector stability is observed at relatively high gains than planar devices such as MSGCs [14,15].

Fig. 4 shows the pulse-height distributions due to TEY and FY signals using the MSGC detector. These signals are attributed to emissions from the Titanium sample originally induced by $8.05 \mathrm{keV}$ photons. The MSGC in this instance was operated in $\mathrm{He} /$ iso- $\mathrm{C}_{4} \mathrm{H}_{10}(75 / 25 \%)$ at a charge gain of approximately $2 \times 10^{3}$. By increasing the repelling grid voltage beyond the sample voltage, it is clear that TEY signals are almost entirely cancelled. For the case shown in Fig. 4 where the sample was held at $V_{\mathrm{d}}=$ $-1500 \mathrm{~V}$, holding the repelling grid at $V_{\mathrm{g}}=-1600 \mathrm{~V}$ was sufficient for this purpose. At that point, the pulse-height distributions, corresponded to the Titanium $K_{\alpha}$ fluorescent photons at $4.51 \mathrm{keV}$ (FY), are evident, which are shown in more detail in Fig. 5.

Since the GEM detector demonstrated much higher charge gains than the MSGC, it was possible to obtain TEY and FY signals at much higher gains whilst using a smaller percentage of iso- $\mathrm{C}_{4} \mathrm{H}_{10}$ quench gas in Helium. Fig. 6 shows pulse height-distributions due to the TEY and FY signals (Titanium sample with $8.05 \mathrm{keV}$ incoming photons) using the GEM detector with $\mathrm{He} /$ iso- $\mathrm{C}_{4} \mathrm{H}_{10}$

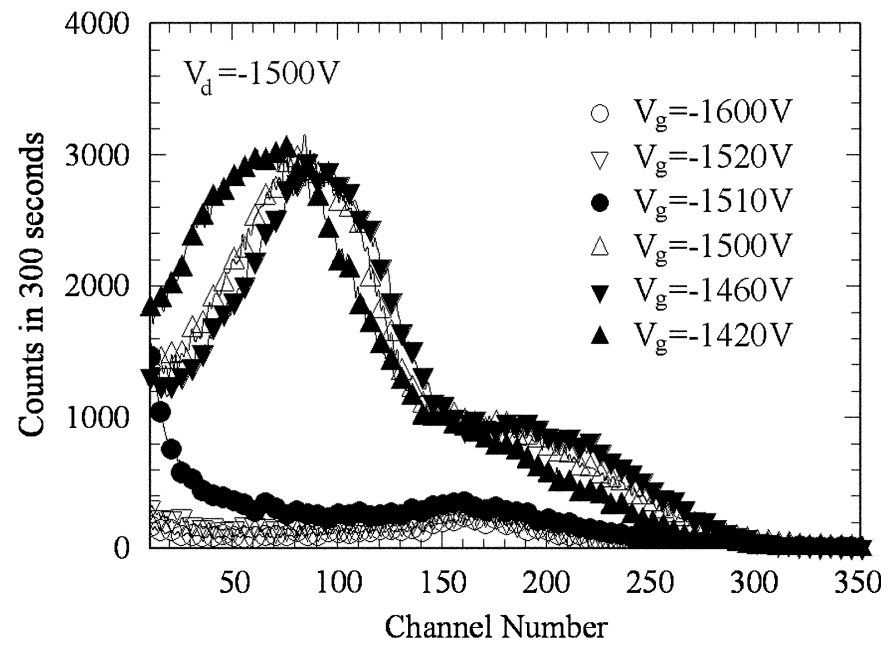

Fig. 4. Pulse-height distributions using the MSGC operated at a charge gain of $2 \times 10^{3}$ with $\mathrm{He} /$ iso- $\mathrm{C}_{4} \mathrm{H}_{10}$ (75/25\%). The Titanium target was held at $V_{\mathrm{d}}=-1500 \mathrm{~V}$ and excited with $8.05 \mathrm{keV}$ X-rays. The upper curves represent TEY and FY signals when the repelling voltage grid $V_{\mathrm{g}} \geqslant-1500 \mathrm{~V}$. The lower curves are due to FY signals when $V_{\mathrm{g}} \leqslant-1500 \mathrm{~V}$.

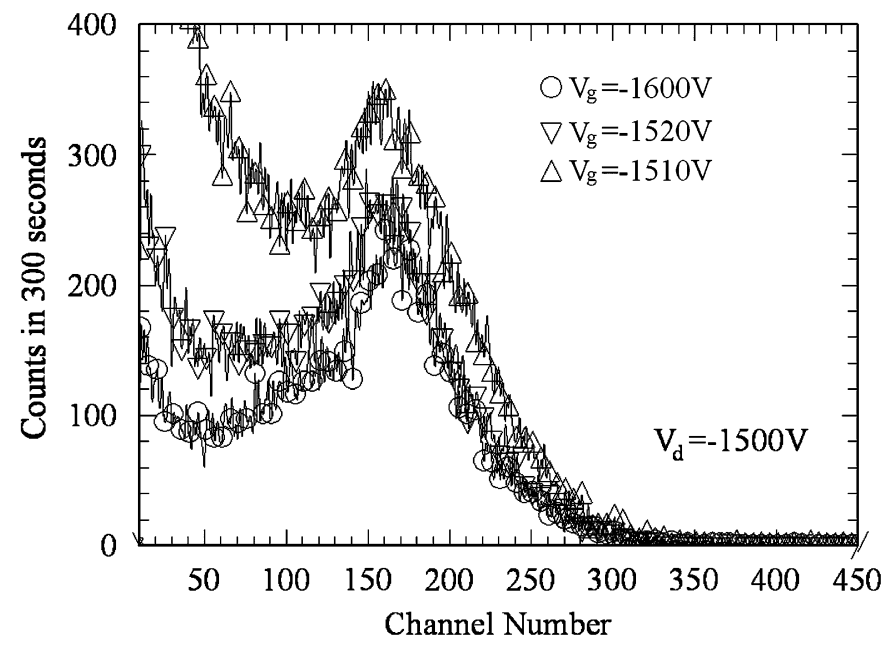

Fig. 5. Detail of pulse-height distributions depicted in Fig. 4, showing only the Titanium $K_{\alpha}$ fluorescent photons at $4.51 \mathrm{keV}$ (FY). 
(85/15\%) when the charge gain was held at $2.8 \times 10^{4}$. The TEY signals were once again eliminated the repelling grid, by a few tens of volts. One of the attractive feature demonstrated by the GEM detector was the superior energy resolution of the FY signals as shown in Fig. 7 (Titanium $K_{\alpha}$ at $4.51 \mathrm{keV}$ ) when compared with FY signals obtained using a MSGC (see Fig. 5).

Fig. 8 shows the FY signals using the GEM detector with the Titanium sample and $8.05 \mathrm{keV}$ incoming photons. Here the gas mixture $\mathrm{He} /$ iso- $\mathrm{C}_{4} \mathrm{H}_{10}(95 / 5 \%)$ was used operating at a gain of approximately $3.2 \times 10^{4}$. The degradation of the X-ray energy resolution is caused by the cumulative effects such as photon feedback, boundary effects and X-ray interactions at the detector chamber walls. The intensity of the photon feedback processes increases with a reducing quench gas concentration due to an increased transparency of spurious UV photons. These photons have sufficient energy to liberate single electrons at, for example, the drift electrodes. The secondary signal is usually very fast and for the present $0.5 \mu$ s shaping amplifier time constant, adds to the primary signal thus giving rise to a pulse height spectrum with a pronounced high energy tail $[16,17]$ as well as a typical exponential tail associated with single electron UV response. The attenuation length of the fluorescence photons also becomes

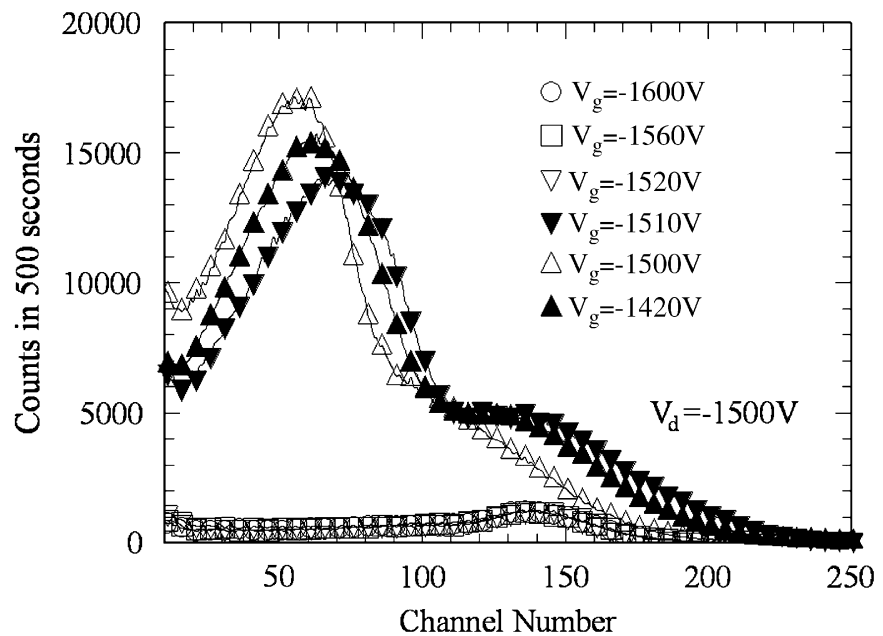

Fig. 6. Pulse-height distributions using the GEM operated at a charge gain of $2.8 \times 10^{4}$ with $\mathrm{He} /$ iso- $\mathrm{C}_{4} \mathrm{H}_{10}(85 / 15 \%)$. The Titanium sample was held at $V_{\mathrm{d}}$ $=-1500 \mathrm{~V}$ and excited with $8.05 \mathrm{keV} \mathrm{X}$-rays. The upper curves represent TEY and FY signals whilst the lower ones are those corresponding to FY signals.

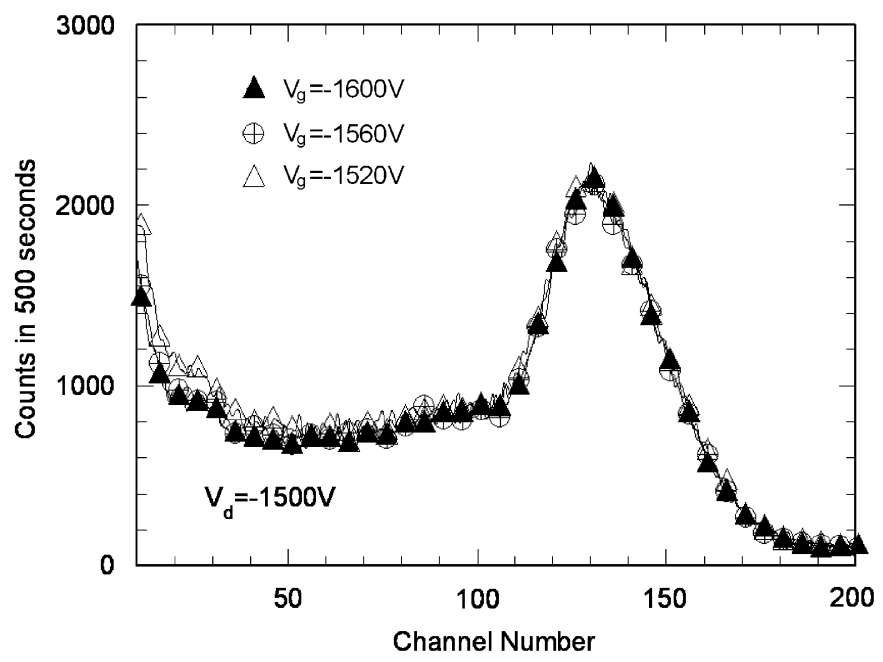

Fig. 7. Detail of pulse-height distributions depicted in Fig. 6, showing only the Titanium $K_{\alpha}$ fluorescent photons at $4.51 \mathrm{keV}(\mathrm{FY})$.

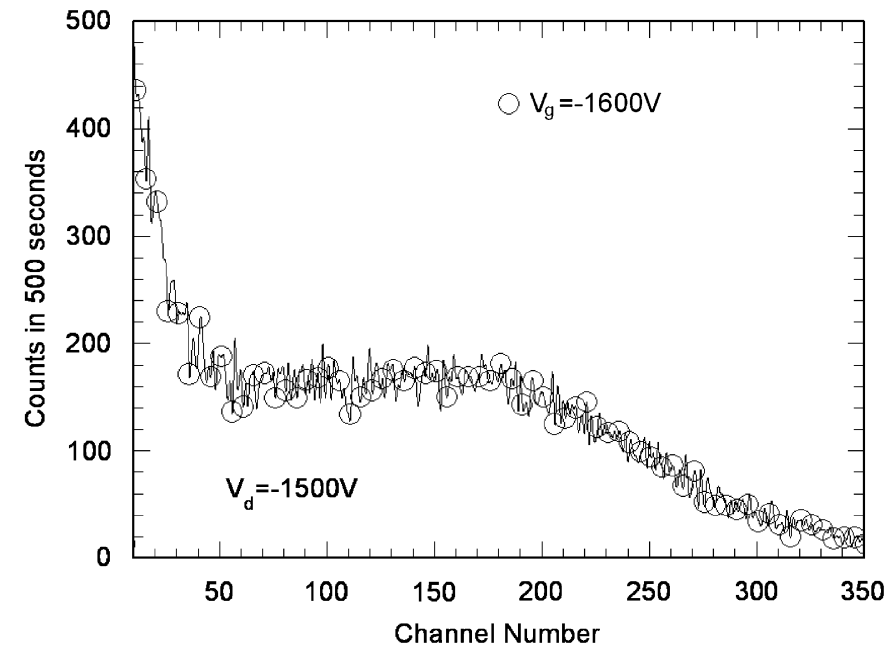

Fig. 8. Pulse-height distributions using the GEM operated at a charge gain of $3.2 \times 10^{4}$ with $\mathrm{He} /$ iso- $_{4} \mathrm{H}_{10}(95 / 5 \%)$.

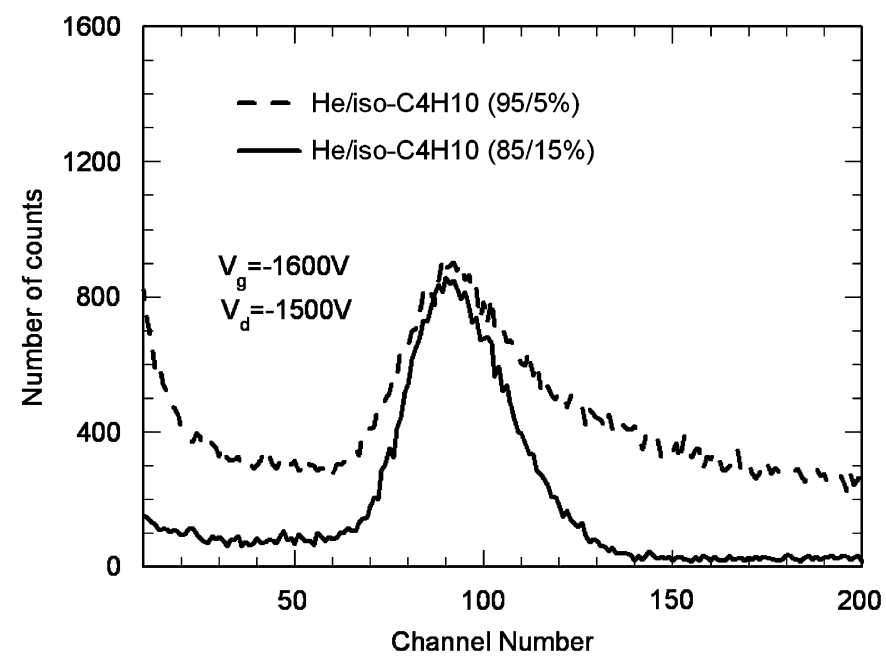

Fig. 9. Pulse-height distributions of the Aluminium $K_{\alpha}$ fluorescence (1.49 keV) photons. The GEM was operated at a charge gain of $2.4 \times 10^{4}$ for both $\mathrm{He} /$ iso- $^{-} \mathrm{C}_{4} \mathrm{H}_{10}$ gas mixtures of $95 / 5 \%$ and $85 / 15 \%$. The electronic gain for these distributions was doubled in relation to the distributions shown in Figs. 7-8.

large with decreasing concentration of the quench gas leading to the detection of partial events at the detector boundaries.

The degradation of the X-ray energy resolution with lower quench gas concentration was cross-checked by observing pulse height distributions due to the fluorescence from an Aluminium target $(1.49 \mathrm{keV})$ using $\mathrm{He} /$ iso- $\mathrm{C}_{4} \mathrm{H}_{10}$ gas mixtures of $85 / 15 \%$ and $95 / 5 \%$, both operated at a charge gain of $2.4 \times 10^{4}$. Whilst near optimum X-ray energy resolution was observed when using the He/iso- $\mathrm{C}_{4} \mathrm{H}_{10}(85 / 15 \%)$ mixture ( $33 \%$ FWHM), much poorer pulse-height spectrum was obtained with the $\mathrm{He} /$ iso- $-\mathrm{C}_{4} \mathrm{H}_{10}$ (95/5\%), as depicted in Fig. 9, where the appearance of events above the $1.49 \mathrm{keV}$ peak, as well as the low energy tail begin to dominate. We should note that the electronic chain gain associated with Fig. 9 was doubled when compared with that used to obtain Figs. 7-8.

\section{Summary and future work}

We have demonstrated that GEM detectors deliver much higher charge gain, at least by an order of magnitude, for He/iso- $\mathrm{C}_{4} \mathrm{H}_{10}$ gas 
mixtures with small quench gas concentration when compared to a MSGC device. This is in converse to the charge gain characteristics of the MSGC in $\mathrm{Ar} /$ iso- $\mathrm{C}_{4} \mathrm{H}_{10}$ gas mixtures. However, when using the MSGC with $\mathrm{He} /$ iso- $\mathrm{C}_{4} \mathrm{H}_{10}$ mixtures, it becomes more prone to the photon feedback effects due to greater transparency of the spurious UV photons and its planar geometry. In contrast, the GEM inherently suppresses the UV photons due to electron avalanche processes taking place within its holes and allows operation in high charge gain regime with reduced quencher concentration. The superior charge gain properties of the GEM detectors makes them potentially useful for XAS experiment at synchrotron beam lines when investigating, for example, transition metals alloys. We have also shown the benefits of using a simple repelling voltage grid to separate the FY signals from the TEY signals.

Further work is in progress to improve the work described in this paper. In particular, the Nickel grid will be substituted by graphite fibres to avoid signal contamination via the TEY and the FY signals associated with Nickel LM transitions. The photon feedback effects lead to instabilities and degradation of the X-ray energy resolution in gaseous detectors and the intensity of these processes increases with increasing charge gains and decreasing quench gas concentration. By utilising a two stage amplification device in the future work, such as a GEM-MIGAS [18-20], higher charge gains are expected with lower iso- $\mathrm{C}_{4} \mathrm{H}_{10}$ concentrations $(\leqslant 5 \%)$ whilst preserving the X-ray energy resolution of the fluorescent photons.

\section{Acknowledgement}

We would like to thank Dr. Barry Dobson of the STFC Daresbury Laboratory for many stimulating discussions on the themes of auger and fluorescence spectroscopy.

\section{References}

[1] A. Oed, Nucl. Instr. and Meth. A 263 (1988) 351.

[2] F. Sauli, Nucl. Instr. and Meth. A 386 (1997) 531.

[3] D.A. Fischer, J. Colbert, J.L. Gland, Rev. Sci. Instrum. 60 (1989) 1596

[4] F. Zaera, D.A. Fischer, S. Shen, J.L. Gland, Surf. Sci. 194 (1988) 205.

[5] T. Rayment, S.L.M. Schroeder, G.D. Moggridge, J.E. Bateman, G.E. Derbyshire, R. Stephenson, Rev. Sci. Instrum. 71 (10) (2000) 3640.

[6] S.L.M. Schroeder, G.D. Moggridge, R.M. Lambert, T.R. Rayment, J. Phys. IV (Colloque) 7 (C2) (1997) 91.

[7] B. Abbey, J.D. Lipp, Z.H. Barber, T. Rayment, Phys. Rev. B Condens. Matter Mater. Phys. 74 (24) (2006) 245408.

[8] B. Abbey, J.D. Lipp, Z.H. Barber, T. Rayment, J. Appl. Phys. 99 (12) (2006) 124914.

[9] J.E. Bateman, G.E. Derbyshire, Ionising particle analyser enabling for example the separation of the fluorescent yield (FY) and the total electron yield (TEY) in EXAFS (extended X-ray absorption fine structure) measurements, United States Patent 20070051898, 2007.

[10] J.E. Bateman, R. Barlow, G.E. Derbyshire, J.A. Mir, R. Stephenson, IEEE Trans. Nucl. Sci. 49 (3) (2002) 894.

[11] A. Sharma, ICFA 16 (1998).

[12] I.K. Bronic, Hoshasen 24 (4) (1998) 101

[13] A. Buzulutskov, L. Shekhtman, A. Bressan, A. Di Mauro, L. Ropelewski, F. Sauli, S. Biaggi, Nuclear Instr. and Meth. Phys. Res. A 433 (1999) 471.

[14] A. Breskin, A. Buzulutskov, R. Chechik, Nucl. Instr. and Meth. Phys. Res. A 483 (2002) 670.

[15] J.E. Bateman, R. Barlow, G.E. Derbyshire, J.A. Mir, R. Stephenson, Nucl. Instr. and Meth. Phys. Res. A 513 (1-2) (2003) 273.

[16] A. Bondar, A. Buzulutskov, L. Shekhtman, R. Snopkov, Y. Tikhonov, Nucl. Instr. and Meth. Phys. Res. A 524 (2004) 130.

[17] A. Aulchenko, A. Bondar, A. Buzulutskov, L. Shekhtman, R. Snopkov, Y. Tikhonov, Nucl. Instr. and Meth. Phys. Res. A 513 (2003) 256.

[18] J.A. Mir, R. Stephenson, N.J. Rhodes, E.M. Schooneveld, H. Natal da Luz, J.F.C.A. Veloso, J.M.F. Dos Santos, C.D.R. Azevedo, Nucl. Instr. and Meth. Phys. Res. A 580 (2007) 1372.

[19] J.A. Mir, R. Stephenson, N.J. Rhodes, E.M. Schooneveld, J.F.C.A. Veloso, J.M.F. Dos Santos, Nucl. Instr. and Meth. Phys. Res. A 573 (2007) 179.

[20] J.A. Mir., G.E. Derbyshire, R. Stephenson, N.J. Rhodes, E.M. Schooneveld, J.F.C.A. Veloso, J.M.F. Dos Santos, N. Spooner, T.B. Lawson, P.K. Lightfoot, IEEE Trans. Nucl. Sci. NS-52 (6) (2005) 2927. 